\title{
Kineto-dynamic Performance Analysis of Vehicle with an Asymmetric Suspension Damper using a Roll-Plane Model
}

\author{
Krishna Prasad Balike*, Subhash Rakheja and Ion Stiharu \\ CONCAVE Research Centre \\ Department of Mechanical \& Industrial Engineering \\ Concordia University \\ 1455 de Maisoneuve West, \\ Montreal, QC, Canada H3G 1M8 \\ E-mail:pras_bk@yahoo.com \\ E-mail: rakheja@alcor.concordia.ca \\ E-mail: istih@alcor.concordia.ca \\ *Corresponding author
}

\begin{abstract}
This paper concerns with the study of influences of damper asymmetry together with the suspension kinematics and tire lateral compliance on the kineto-dynamic responses of a roll-plane half-car model. Such coupled analysis of kinematic and vertical and roll dynamic measures of the vehicle involving asymmetric suspension dampers has not been reported in the literature. A 4-DOF, roll-plane model of the road vehicle employing a double wishbone type suspension comprising a strut with linear spring and asymmetric damper is formulated for the analyses. The influences of asymmetric dampers are studied by comparing the sprung mass vertical acceleration, chassis roll angle and the dynamic tire forces, and the left- and right tires camber angle variation responses of the model with asymmetric dampers with those of the model with an equivalent linear damper under bump and pothole inputs. The influences of damper asymmetry are also investigated under rounded-step lateral excitations. The results of the study suggested that the responses are complex functions of damper asymmetry ratio, defined as the ratio of damping coefficient in rebound to that in compression, vehicle forward speed and type of input. The study further suggests that a very low compression mode damping is undesirable from both ride and handling dynamic perspectives.
\end{abstract}

Keywords: Automotive suspension, Kineto-dynamic model, damper asymmetry, suspension kinematics, tire lateral compliance, vehicle ride and handling analysis.

Reference to this paper should be made as follows: Balike, K.P., Rakheja, S., and Stiharu, I. (20xx) 'Kineto-dynamic performance analysis of vehicle with an asymmetric suspension damper using a roll-plane model', Int. J. Vehicle Performance, Vol. xx, Nos. xx, pp. xx-xx. 
Biographical notes: K.P. Balike, $\mathrm{Ph} \mathrm{D}$ is a Senior Analyst in Pratt \& Whitney Canada and working on Aero-Engine component dynamics. He obtained his $\mathrm{Ph} \mathrm{D}$ degree from Concordia University, Canada. During his $\mathrm{Ph} \mathrm{D}$, the area of research included vehicle dynamics, suspension component synthesis and analysis, kinematics of suspension and influence of kinematics on dynamics of the vehicle.

Subhash Rakheja, Ph D, Fellow of ASME, is a Professor of Mechanical Engineering and Vehicular Ergodynamics Research Chair at the CONcordia Centre for Advanced Vehicle Engineering (CONCAVE), Concordia University, Montreal, Canada.

Ion Stiharu, $\mathrm{PhD}$, is a Professor in Department of Mechanical and Industrial Engineering, Concordia University in Canada. He is the Director of CONcordia Centre for Advanced Vehicle Engineering (CONCAVE) at Concordia University.

\section{Introduction}

The ride and handling performance measures of a vehicle are influenced by the suspension geometry and forcing elements including suspension spring and damper in a significant and conflicting manner. The kinematic response measures such as bump and roll camber angles impose additional conflicting design requirements on the suspension components [Milliken, 1995 and Dixon, 2007]. While suspension designs invariably include asymmetric damping in compression and rebound, it has been identified that the design guidance for such asymmetry has not been explicitly defined in the literature [Dixon, 2007]. This is, in part, attributed to the limited understanding of influences of damper asymmetry on the kineto-dynamic performance measures. Recent studies based on a two-DOF kineto-dynamic quarter-car model have shown strong couplings between the kinematic and vertical dynamic responses, which further depend upon the suspension damping asymmetry in a complex manner [Balike et al., 2010 (ref 3) and Balike et al., 2011 (ref 4)]. The complex dependency of damper asymmetry on the dynamic and kinematic responses would be expected to increase many folds, when coupled vertical and roll motions of the chassis are considered.

The suspension damping properties and their effects on various vehicle performance measures have been extensively investigated under different inputs, including the contributions due to gas spring, bushings compliance, and temperature and hysteresis effects [Anderson and Fan, 1990, Duym et al., 1997, Basso, 1998, Gacka and Doherty, 2006, Simms and Crolla, 2002]. These studies have employed different vehicle models, excitations and different performance measures, while the majority of them have ignored the damping asymmetry. The reported results thus do not permit the design guidance for damping asymmetry, which has been limited to a general rule of thumb suggesting that a rebound to compression damping ratio in the order of 2 or 3 would reduce the force transmitted to the sprung mass, while negotiating a bump [Milliken, 1995, Gillespie, 1992]. 
A few studies have suggested that damping asymmetry causes suspension 'packing or jacking down' [Warner and Rakheja, 1996 and Rajalingham and Rakheja, 2003], which is apparently change in the ride height and is shown to be dependent upon the low speed compression and rebound damping coefficients of the damper. Simms and Crolla [2002] showed the presence of this drift under random road excitations using a quartercar model incorporating hysteresis model of a damper. The simulation results obtained with an asymmetric damper with relatively higher rebound damping revealed large offset in the suspension rattle space response compared to that with the linear damping. Verros et al. [2000] investigated the transient response of a single-degree-of-freedom (DOF) quarter-car model with single-stage asymmetric dampers under pothole excitations.

Optimal suspension damper synthesis has been considered as a very challenging task, as is evident from the number of studies reported on damper synthesis [Balike et al., 2010 (ref 3), Balike et al., 2011 (ref 4), Fukushima et al., 1983, Gobbi and Mastinu, 2001, Alkhatib et al., 2004, Verros et al., 2005, Georgiou et al., 2007, Gobbi et al., 1999, Bruulsema and McPhee, 2002, He and McPhee, 2007, Georgiou, and Natsiavas, 2009]. Majority of these studies have considered simple analytical vehicle models (quarter, half or full vehicle) for the selection of damping coefficients and analysis of suspension damping properties on the selected vehicle performance measures [Dixon, 2007, Balike et al., 2010 (ref 3), Fukushima et al., 1983, Gobbi and Mastinu, 2001, Alkhatib et al., 2004, Verros et al., 2005, Georgiou et al., 2007]. Many other studies have also employed complex multibody dynamic models, developed in platforms such as ADAMS, for the selection of optimal damping coefficients [Gobbi et al., 1999, Bruulsema and McPhee, 2002, He and McPhee, 2007, Georgiou, and Natsiavas, 2009]. The identification of most adequate performance measures during damper synthesis is considered to be as complicated as the selection of optimal damping coefficients itself [Bruulsema and McPhee, 2002]. This can be attributed to the limited understanding of couplings among various response measures, particularly when an asymmetric damper is employed in the suspension. For example, recent studies using kineto-dynamic quarter-car model incorporating linkage kinematics of a double wishbone suspension and tire lateral compliance have shown the effects of damper jacking on the kinematic response measures (such as camber variation) [Balike et al., 2011 (ref 3)], which were ignored in the previous studies. These studies showed that the change in ride height due to damper asymmetry would also change the bump camber angle responses under deterministic bump/pothole or random road excitations. The studies could not, however, report the influences of damping asymmetry on the roll camber responses due to limitations of simpler quarter-car model. A systematic study of influences of damper asymmetry on various performance measures related to ride and handling dynamics is desirable prior to optimal synthesis of an asymmetric damper.

The levels of required modeling complexity in developing vehicle models for dynamic analyses have long been debated. Although complex multi-body dynamic models are known to achieve greater accuracy, many researchers have put forward the view that typical industry-used vehicle models are too complex and inefficient as design and concept analysis tools [Blundell, 1999, Sharp 1991]. Sharp [1991] suggested that an ideal model should possess minimum complexity and be capable of solving the concerned problems with an acceptable accuracy. Roll-plane dynamic models have effectively been used to study the influences of suspension damping properties on the ride dynamic and the essential handling behavior of the vehicle [Su, 1990]. A kinetodynamic roll-plane model which incorporates the linkage kinematics is necessary for 
studying the influences of damping asymmetry on the suspension kinematic measures and vehicle dynamic responses with a reasonable accuracy.

This paper concerns with the influences of damper asymmetry together with the suspension kinematics and tire lateral compliances on the kineto-dynamic responses of a roll-plane half-car model under vertical and lateral inputs. Unlike a few previous studies those considered effects of suspension kinematics and tire lateral compliances employing quarter-car model, this study is more comprehensive with the inclusion of half-car model. A 4-DOF, half-car kineto-dynamic model of the road vehicle employing a double wishbone type suspension comprising a strut with linear spring and asymmetric damper is formulated for the analyses. The displacement matrix method is employed to derive kinematic formulations, while the Lagrange's method is used to formulate the dynamic model. The influences of asymmetric dampers are studied by comparing the sprung-mass vertical acceleration, chassis roll angle, normalized load transfer, and the left- and right wheel camber angle variation responses of the model with asymmetric dampers with those of the model with an equivalent linear damper. The responses of the model are further studied under lateral acceleration inputs to the sprung mass corresponding to steering inputs or wind gust.

\section{Development of roll-plane kineto-dynamic vehicle model}

The in-plane 4-DOF half-car kineto-dynamic model comprising double wishbone type of suspension, employed in this study is illustrated in Fig. 1. The model comprises the sprung and two unsprung masses (left and right wheel assemblies), while the unsprung masses are assumed to be connected with the chassis through massless control arms. Although control arm inertia is expected to cause a small variation to the dynamics of the system, its influence is considered insignificant, particularly when the study is focused on the comparative performance of different dampers. Each unsprung mass is assumed to be lumped at the center of gravity (cg) of the wheel assembly. The tire is modeled as a combination of a vertical linear spring and a viscous damper, while the lateral compliance of the tire is represented by a lateral linear stiffness, as shown in the figure. The chassis and suspension kinematics are formulated considering the chassis, suspension linkages and the wheel spindle as rigid bodies. The model is formulated assuming vertical $\left(z_{s}\right)$ and roll $\left(\phi_{s)}\right.$ displacements of the sprung mass, and left and right wheels vertical displacements $\left(z_{u L}\right.$ and $\left.z_{u R}\right)$ as the generalized coordinates. The rotation of the chassis is assumed to occur about the roll center $R c$, as shown in Fig. 1.

Figure 1. Roll-plane kineto-dynamic model of a vehicle with double wishbone type of suspension.

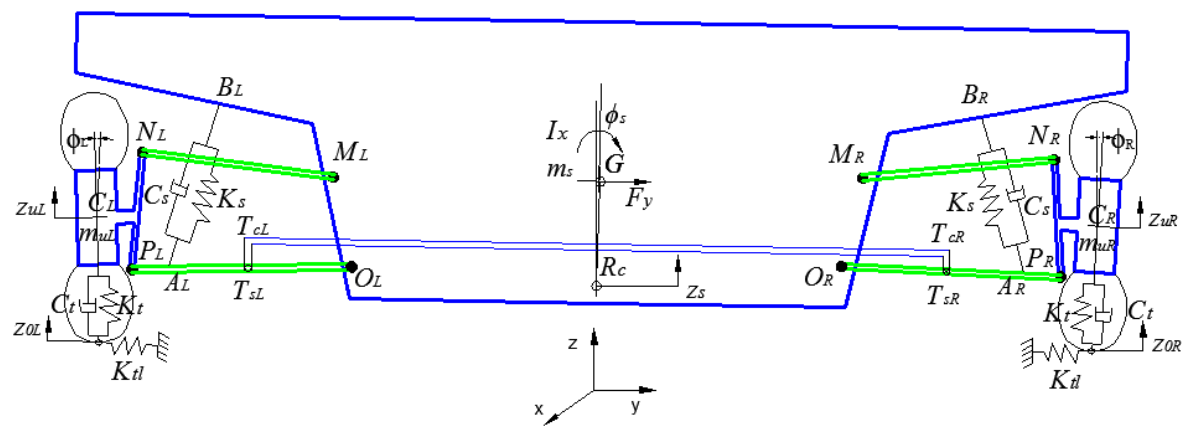




\subsection{Kinematics of the Chassis and the Suspensions}

A chassis kinematic model is formulated in order to evaluate instantaneous positions of suspension joints on the chassis under chassis rotation and/or vertical motions. The suspension kinematic responses are subsequently determined from coordinates of the linkage joints. A fixed coordinate system is considered with its origin located in the ground, while the sprung mass vertical and rotational displacements are considered about the roll center, $R c$, of the vehicle body. The initial $\left(R c_{y 0}, R c_{z 0}\right)$ and instantaneous $\left(R c_{y}\right.$, $R c_{z}$ ) coordinates of the roll center under a finite displacement of the chassis are related through a displacement matrix [Suh and Radcliffe, 1978 and Balike et al., 2010 (ref 27)]. The $y$ - and $z$ - coordinates of chassis-suspension joints, $M_{R}, O_{R}, M_{L}$, and $O_{L}$, shown in the Fig. 1 are determined using the displacement matrix, as presented in [Balike et al., 2010 (ref 27)]. The resulting expressions for the chassis joint coordinates are written as:

$$
\begin{aligned}
& M_{k y}=a_{11}\left(M_{k y 0}-R c_{y 0}\right)+a_{12}\left(M_{k z 0}-R c_{z 0}\right)+R c_{y} \\
& M_{k z}=a_{21}\left(M_{k y 0}-R c_{y 0}\right)+a_{22}\left(M_{k z 0}-R c_{z 0}\right)+R c_{z} \\
& O_{k y}=a_{11}\left(O_{k y 0}-R c_{y 0}\right)+a_{12}\left(O_{k z 0}-R c_{z 0}\right)+R c_{y} \\
& O_{k z}=a_{21}\left(O_{k y 0}-R c_{y 0}\right)+a_{22}\left(O_{k z 0}-R c_{z 0}\right)+R c_{z} \quad k=R, L
\end{aligned}
$$

where $a_{11}=a_{22}=\cos \phi_{s}$ and $a_{12}=-a_{21}=\sin \phi_{s}$, with $\phi_{s}$ being the vehicle body rotation about the roll center. The leading subscripts ' $R$ ' and ' $L$ ' in Eq (1) refer to the right and left chassis joints, respectively, while the second subscripts ' $y$ ' and ' $z$ ' represent the lateral and vertical axes, respectively. The final subscript ' $O$ ' refers to the initial coordinate of the joint. Furthermore, the instantaneous coordinates of roll center $\left(R c_{y}, R c_{z}\right)$ are obtained from $R c_{y}=R c_{y 0}$; and $R c_{z}=R c_{z 0}+z_{s}$, respectively. The above equation can be solved to obtain instantaneous coordinates of the chassis-linkage joints for a given chassis rotation $\phi_{s}$ about the roll center and/or a vertical displacement of the chassis, $z_{s}$.

The kinematic analysis of the suspension links is performed in a manner similar to that of the chassis employing displacement matrices defined for the right and left wheel spindles. The instantaneous coordinates of the suspension-spindle joints $\left(N_{R}, P_{R}, N_{L}\right.$ and $P_{L}$ ), following a wheel spindle displacement, are expressed using the displacement matrices written in terms of initial $\left(C_{k y 0}, C_{k z 0}\right)$ and instantaneous $\left(C_{k y}, C_{k z}\right)$ coordinates of the right wheel centers, $C_{k}$. The methodology of deriving these kinematic expressions is discussed in more detail in [Balike et al., 2010 (ref 27)]. The resulting formulation consists of 8 equations with a total of 12 unknown parameters corresponding to the rightand left-wheel center displacements $z_{u R}$ and $z_{u L}$, namely: the $y$ and $z$ coordinates of joints $N_{R}, P_{R}, N_{L}$ and $P_{L}$; the $y$ coordinates of the wheel centers $C_{R}$ and $C_{L}$; and the right- and left-wheel camber angles $\phi_{R}$ and $\phi_{L}$. The coordinate equations are thus solved in conjunction with the constraint equations, which for a planar double wishbone suspension may be formulated considering the constant control arm lengths. The expressions for the instantaneous coordinates of the suspension joints, $N_{R}, P_{R}, N_{L}$ and $P_{L}$, obtained from the displacement matrices together with the constraint equations thus yields a system of 12 non-linear equations, given by [Balike et al., 2010 (ref 27)]:

$$
\begin{aligned}
& N_{k y}=a_{11 k}\left(N_{k y 0}-C_{k y 0}\right)+a_{12 k}\left(N_{k z 0}-C_{k z 0}\right)+C_{k y} \\
& N_{k z}=a_{21 k}\left(N_{k y 0}-C_{k y 0}\right)+a_{22 k}\left(N_{k z 0}-C_{k z 0}\right)+C_{k z 0}+z_{u k}
\end{aligned}
$$




$$
\begin{aligned}
& P_{k y}=a_{11 k}\left(P_{k y 0}-C_{k y 0}\right)+a_{12 k}\left(P_{k z 0}-C_{k z 0}\right)+C_{k y} \\
& P_{k z}=a_{21 k}\left(P_{k y 0}-C_{k y 0}\right)+a_{22 k}\left(P_{k z 0}-C_{k z 0}\right)+C_{k z 0}+z_{u k} \\
& \left(N_{k y}-M_{k y}\right)^{2}+\left(N_{k z}-M_{k z}\right)^{2}=l_{M N k}^{2} \\
& \left(P_{k y}-O_{k y}\right)^{2}+\left(P_{k y}-O_{k y}\right)^{2}=l_{O P k}^{2} \quad k=R, L
\end{aligned}
$$

where $a_{11 k}=a_{22 k}=\cos \phi_{k}$ and $a_{12 k}=-a_{21 k}=\sin \phi_{k}, k=R, L$. In the above equation, $l_{M N k}$ and $l_{O P k}$ $(k=R, L)$ are the lengths of upper and lower control arms, respectively.

Equations (1) and (2) can be simultaneously solved to obtain kinematic responses of the suspension for given vertical displacements of the left and/or right wheels, and/or vertical and roll displacements of the chassis. Closed form solutions of the unknowns in terms of generalized coordinates would be desirable in order to correlate the kinematic relations to the dynamic responses, which may be quite complex. A linear system of kinematic relations for the chassis joints could be achieved using small angles assumptions, such that $a_{11}=a_{22} \approx 1$; and $a_{21}=-a_{21} \approx \phi_{s}$ :

$$
\begin{aligned}
& M_{k y}=M_{k y 0}+\phi_{s}\left(M_{k z 0}-R c_{z 0}\right) ; \quad M_{k z}=-\phi_{s}\left(M_{k y 0}-R c_{y 0}\right)+M_{k z 0}+z_{s} \\
& O_{k y}=O_{k y 0}+\phi_{s}\left(O_{k z 0}-R c_{z 0}\right) ; O_{k z}=-\phi_{s}\left(O_{k y 0}-R c_{y 0}\right)+O_{k z 0}+z_{s}
\end{aligned}
$$

Similarly, the small angle assumptions in the kinematic equations of the suspension linkages yield $a_{11 k}=a_{22 k} \approx 1$; and $a_{12 k}=-a_{21 k} \approx \phi_{k}$. The small angle assumptions in conjunction with the first-order Taylor series approximations of the constraint equations yield the kinematic relations in the linear form as:

$$
\begin{aligned}
& N_{k y}-\phi_{k}\left(N_{k z 0}-C_{k z 0}\right)-C_{k y}=\left(N_{k y 0}-C_{k y 0}\right) \\
& N_{k z}+\phi_{k}\left(N_{k y 0}-C_{k y 0}\right)=N_{k z 0}+z_{u k} \\
& P_{k y}-\phi_{k}\left(P_{k z 0}-C_{k z 0}\right)-C_{k y}=\left(P_{k z 0}-C_{k y 0}\right) \\
& P_{k z}+\phi_{k}\left(P_{k y 0}-C_{k y 0}\right)=P_{k z 0}+z_{u k} \\
& \left(N_{k y 0}+M_{k y 0}-2 M_{k y}\right) N_{k y}+\left(N_{k z 0}+M_{k z 0}-2 M_{k z}\right) N_{k z} \\
& \quad=N_{k y 0}^{2}+M_{k y 0}^{2}+N_{k z 0}^{2}+M_{k z 0}^{2}-\left(N_{k y 0}+M_{k y 0}\right) M_{k y}-\left(N_{k z 0}+M_{k z 0}\right) M_{k z} \\
& \left(P_{k y 0}+O_{k y 0}-2 O_{k y}\right) P_{k y}+\left(P_{k z 0}+O_{k z 0}-2 O_{k z}\right) P_{k z} \\
& \quad=P_{k y 0}^{2}+O_{k y 0}^{2}+P_{k z 0}^{2}+O_{k z 0}^{2}-\left(P_{k y 0}+O_{k y 0}\right) O_{k y}-\left(N_{k z 0}+M_{k z 0}\right) O_{k z}
\end{aligned}
$$

Equation (4) is solved to obtain expressions for the kinematic responses of the leftand right $(k=L$ and $R$ ) suspensions, which include the instantaneous coordinates of the joints and the wheel camber angles, in terms of the generalized coordinates, and are shown in Appendix I.

The restoring force developed by each strut is related to the change in the strut length, $\Delta l_{k}$ given by: 


$$
\Delta l_{k}=l_{s 0}-\left[\left(A_{k y}-B_{k y}\right)^{2}+\left(A_{k z}-B_{k z}\right)^{2}\right]^{1 / 2}
$$

where $l_{\mathrm{s} 0}$ is the initial strut length, assumed to be identical for the left and right struts. In the above expression, $\left(A_{k y}, A_{k z}\right)$ and $\left(B_{k y}, B_{k z}\right)$ are the instantaneous coordinates of the lower and upper strut mounts, which can be obtained from the kinematics of the chassis and suspension, as:

$$
\begin{aligned}
& A_{k y}=O_{k y}+\frac{l_{O A}}{l_{O P}}\left(P_{k y}-O_{k y}\right) \quad A_{k z}=O_{k z}+\frac{l_{O A}}{l_{O P}}\left(P_{k z}-O_{k z}\right) \\
& B_{k y}=B_{k y 0}+\phi_{s}\left(B_{k z 0}-R c_{z 0}\right) ; \quad B_{k z}=-\phi_{s}\left(B_{k y 0}-R c_{y 0}\right)+B_{k z 0}+z_{s}
\end{aligned}
$$

where $l_{O A}$ and $l_{O P}$ are the distances of the joints $A_{R}$ and $P_{R}$ from point $O_{R}$ (or $A_{L}$ and $P_{L}$ from point $O_{L}$ ). The deflection rates of left- and right suspension struts are subsequently estimated from the time derivatives of the displacement expressions in $\mathrm{Eq}$ (6).

\subsection{Kinematics of antiroll bar}

Anti-roll bars are invariably employed in vehicle suspensions in order to enhance roll stiffness and to reduce dynamic load transfers. An anti-roll bar couples vertical motions of the right and left wheels, and develops a resisting roll moment under chassis roll or differential wheel motions. The kinematic motion of a torsion bar thus involves spatial kinematic analysis, as illustrated in Fig. 2. The torsion bar is assumed to be coupled to the chassis at points $T c_{L}$ and $T c_{R}$, and to the lower control arms at the points $T s_{L}$ and $T s_{R}$, respectively. The instantaneous $z$ - coordinates of the chassis mounting points $T_{c}(k=L, R)$ are estimated from the kinematics of the chassis as:

$T c_{k z}=-\phi_{s}\left(T c_{k y 0}-R c_{y 0}\right)+T c_{k z 0}+z_{s}$

where $T c_{k y 0}$ and $T c_{k z 0}$ are the initial $y$ and $z$ coordinates, respectively, of $T c_{L}$ and $T c_{R}$. The torsional deformation of the torsion bar $\theta_{T}$ is determined from the changes in the coordinates of the mounting points, as:

$\theta_{T}=\frac{1}{L_{T}}\left[\left(T c_{L z}-T s_{L z}\right)+\left(T c_{R z}-T s_{R z}\right)\right]-2 \theta_{T 0}$

where $L_{T}$ is the effective length of the torsion bar between attachment points $T s_{R}$ and $T c_{R}$, and $\theta_{T O}$ is the initial deformation angle of the torsion bar arm with respect to the horizontal axis of the reference coordinate system. The subscripts ' $z$ ' and ' $O$ ' are used to represent the $z$-coordinates and the initial coordinates, respectively. The $z$-coordinates of the torsion bar mounting points at the lower control arms $T s_{R z}$ and $T s_{L z}$, are obtained from the linkage kinematics, as:

$T s_{k z}=O_{k z}+\frac{l_{O T s}}{l_{O P}}\left(P_{k z}-O_{k z}\right) \quad k=R, L$ 
where $l_{O T S}$ is the length of the lower control arm between the torsion bar mounting point, $T s_{R}$ and the chassis joint $O_{R}$.

Figure 2: Kinematics of the torsion bar

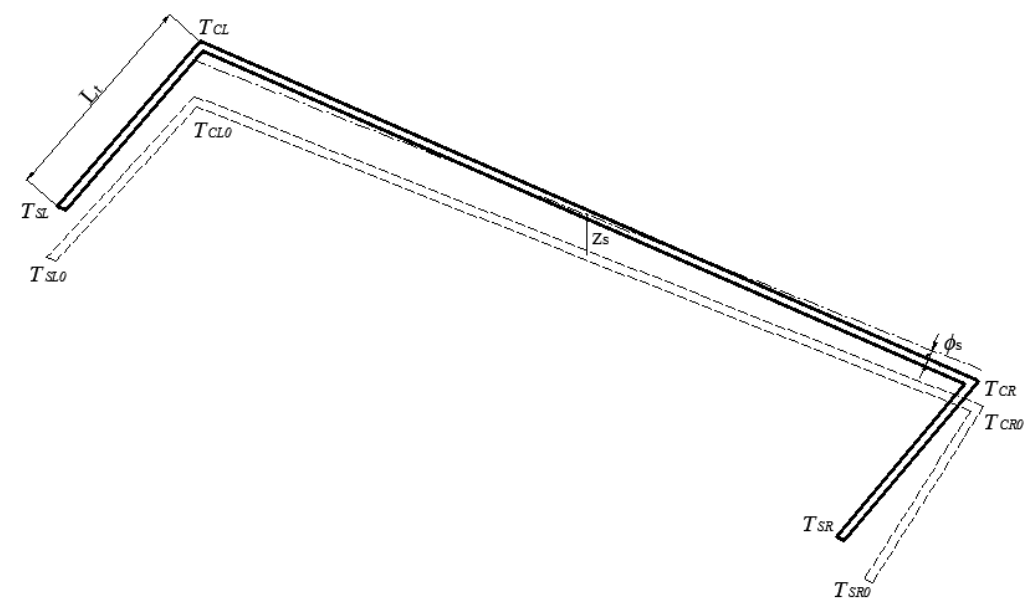

\subsection{Kineto-dynamic Analysis of the Half-Car Model}

The equations of dynamic motion of the kineto-dynamic half-vehicle system are derived using Lagrange's method. The kinetic energy $(T)$ of the system is formulated as:

$$
\begin{aligned}
T= & \frac{1}{2} m_{\mathrm{s}}\left(\dot{z}_{\mathrm{s}}^{2}+\dot{y}_{\mathrm{s}}^{2}\right)+\frac{1}{2}\left(I_{x}+\left(G_{z 0}-R c_{z 0}\right)^{2} m_{s}\right) \dot{\phi}_{\mathrm{s}}^{2} \\
& +\frac{1}{2} \sum_{k=R, L} m_{u k}\left(\dot{y}_{u k}^{2}+\dot{z}_{u k}^{2}\right)+\frac{1}{2} \sum_{k=R, L} I_{u k x} \dot{\phi}_{k}^{2}
\end{aligned}
$$

where $m_{s}, m_{u R}$ and $m_{u L}$ are sprung mass, and right- and left unsprung masses, respectively. In the above expression, $I_{x}$ and $I_{u k x}(k=R, L)$ are the mass moment of inertia of the chassis and the right- and left wheel spindles about the $x$-axis, respectively. In Eq (10), $z_{s}^{\prime}$ and $y_{s}^{\prime}$ are instantaneous coordinates of the chassis centre of gravity. The potential energy of the system is expressed as:

$U=\frac{1}{2} \sum_{k=R, L}\left(K_{\mathrm{s}}\left(\Delta l_{k}\right)^{2}+K_{t}\left(\Delta z_{t k}\right)^{2}+K_{t l}\left(y_{u k}-\phi_{k} R_{k}\right)^{2}\right)+\frac{1}{2} K_{t b} \theta_{T}^{2}$

where $K_{s}$ is the suspension spring rate, $K_{t}$ is the equivalent tire vertical rate, $K_{t b}$ is the linear stiffness of the torsion bar, $K_{t l}$ is the tire lateral stiffness and $R_{k}$ is the effective radius of wheel $k$. Moreover, $\Delta l_{k}$ are the right- and left suspension spring deflections, as described in $\mathrm{Eq}$ (5), and $\Delta z_{t k}$ are the right- and left tire deflections. The total energy dissipated by the system, attributed to the linear strut and tire damping, can be derived as:

$D=\frac{1}{2} \sum_{k=R, L} C_{s}\left(\Delta \dot{l}_{k}\right)^{2}+\frac{1}{2} \sum_{k=R, L} C_{t}\left(\Delta \dot{z}_{t k}\right)^{2}$ 
where $C_{s}$ and $C_{t}$ are the viscous damping coefficients of the strut and the tire, respectively, and $\Delta i_{k}$ denotes the time derivatives of the right- and left strut deflections, and $\Delta \dot{l}_{t k}$ are the rates of right- and left tires deflections.

The sprung mass of a vehicle experiences centrifugal acceleration during cornering maneuvers or due to crosswinds. The centrifugal force due to lateral acceleration of the vehicle encountered during turning maneuvers, denoted as $F_{y}$ in the Fig.1, can be estimated from $F_{y}=m_{s} a_{y}$, where $a_{y}$ is the lateral acceleration, which is estimated from $a_{y}=V^{2} / R_{\text {turn }}$, assuming steady-state condition. The model is thus formulated to include the capability to evaluate in-part the handling behavior of the vehicle.

The equations of motion for the kineto-dynamic model are formulated from the kinetic $(T)$, potential $(U)$ and dissipative $(D)$ energy functions described in Eqs (10) to (12). Assuming negligible contributions due to higher order derivative terms, the equations of motion are obtained as:

$$
\begin{gathered}
m_{\mathrm{s}} \ddot{z}_{s}+\sum_{k=R, L} m_{u k}\left(\ddot{y}_{u k} \frac{\partial \dot{y}_{u k}}{\partial \dot{z}_{\mathrm{s}}}\right) \\
+\sum_{k=R, L} I_{u x k}\left(\ddot{\phi}_{k} \frac{\partial \dot{\phi}_{k}}{\partial \dot{z}_{\mathrm{s}}}\right) \\
+\sum_{k=R, L}\left(f_{s s k}+f_{d s k}+f_{t l s k}\right)+f_{t b s}=-m_{s} g \\
\left(I_{x}+\left(G_{z 0}-R c_{z 0}\right)^{2} m_{s}\right) \ddot{\phi}_{s}+\sum_{k=R, L} m_{u k}\left(\ddot{y}_{u k} \frac{\partial \dot{y}_{u k}}{\partial \dot{\phi}_{s}}\right)+\sum_{k=R, L} I_{u x k}\left(\ddot{\phi}_{k} \frac{\partial \dot{\phi}_{k}}{\partial \dot{\phi}_{s}}\right) \\
+\sum_{k=R, L}\left(T_{s s k}+T_{d s k}+T_{t l s k}\right)+T_{t b s}=\left(G_{z}-R c_{z}\right)\left(F_{y}+m_{s} g \phi_{s}\right) \\
m_{u R} \ddot{z}_{u R}+m_{u R}\left(\ddot{y}_{u R} \frac{\partial \dot{y}_{u R}}{\partial \dot{z}_{u R}}\right)+I_{u x R}\left(\ddot{\phi}_{R} \frac{\partial \dot{\phi}_{R}}{\partial \dot{z}_{u R}}\right) \\
+f_{s u R}+f_{t l u R}+f_{d u R}+f_{t R}+f_{t b u R}=-m_{u R} g \\
m_{u L} \ddot{z}_{u L}+m_{u L}\left(\ddot{y}_{u L} \frac{\partial \dot{y}_{u L}}{\partial \dot{z}_{u L}}\right)+I_{u x L}\left(\ddot{\phi}_{L} \frac{\partial \dot{\phi}_{u L}}{\partial \dot{z}_{u L}}\right) \\
+f_{s u L}+f_{t l u L}+f_{d u L}+f_{t L}+f_{t b u L}=-m_{u L} g
\end{gathered}
$$

where $f_{\text {ssk }}$ and $f_{\text {suk }}(k=R, L)$ are the right- and left- suspension spring forces acting on the sprung and unsprung masses, respectively, $f_{d s k}$ and $f_{d u k}$ are the right- and left- damping forces acting on the sprung and unsprung masses, respectively, and $T_{s s k}$ and $T_{d s k}$ are the moments due to right- and left- suspension spring and damping forces, respectively. In Eq (13), $f_{t l s k}$ and $f_{t l u k}$ are the vertical tire forces acting on the sprung and unsprung masses, respectively, and $T_{t l s k}$ are the moments imposed on the sprung mass due to the right- and left- tire lateral compliance, respectively. Moreover, $f_{t k}$ are the tire forces, and $f_{t b s \text {, and }} f_{t b u k}$ are the forces transmitted to the sprung and unsprung masses, and $T_{t b s}$ is the torque transmitted to the sprung mass due to the torsion bar. Assuming linear spring rates, the suspension spring forces $f_{s s k}$ and $f_{\text {suk }}$ are related to $\Delta l_{k}$, as: 


$$
f_{s s k}=K_{\mathrm{s}} \Delta l_{\mathrm{k}} \frac{\partial\left(\Delta l_{k}\right)}{\partial z_{\mathrm{s}}} \quad ; \text { and } \quad f_{s u k}=K_{s} \Delta l_{k} \frac{\partial\left(\Delta l_{k}\right)}{\partial z_{u k}} \quad k=R, L
$$

The torque imposed on the sprung mass due to the right- and left- suspension springs, $T_{s s k}$ is related to $\Delta l_{k}$ and chassis roll, as:

$T_{s s k}=K_{s} \Delta l_{k} \frac{\partial\left(\Delta l_{k}\right)}{\partial \phi_{s}}$

$$
k=R, L
$$

The vertical forces due to the torsion bar exerted on the sprung mass, and left- and right unsprung masses, $f_{t b s}$ and $f_{t b u k}$, and the torque on the sprung mass, $T_{t b s}$, are obtained from:

$$
f_{t b s}=K_{t b} \theta_{T} \frac{\partial \theta_{T}}{\partial z_{\mathrm{s}}} f_{t b u k}=K_{t b} \theta_{T} \frac{\partial \theta_{T}}{\partial z_{u k}} ; \text { and } \quad T_{t b s}=K_{t b} \theta_{T} \frac{\partial \theta_{T}}{\partial \phi_{\mathrm{s}}} \quad k=R, L
$$

The nonlinearity associated with potential loss of contact between the ground and the tire (wheel hop) is also incorporated in the kineto-dynamic model. The tire forces $\left(f_{t k}\right)$, and the forces and moments due to the tire lateral compliance $f_{t l s k}, f_{t l u k}$ and $T_{t l s k}$ are formulated considering four different possible conditions; namely: (i) both the tires are in contact with the ground $\left(z_{u L^{-}} z_{0 L}<\delta_{u}\right.$ and $\left.z_{u R^{-}} z_{O R}<\delta_{u}\right)$, where $\delta_{u}$ is the static tire deflection; (ii) left wheel in contact with the ground, while the right wheel loses the ground contact $\left(z_{u L^{-}}\right.$ $z_{0 L}<\delta_{u}$ and $z_{u R^{-}} z_{0 R} \geq \delta_{u}$ ); (iii) right wheel is in contact with the ground, while the left wheel loses the ground contact $\left(z_{u L}-z_{O L} \geq \delta_{u}\right.$ and $\left.z_{u R^{-}} z_{0 R}<\delta_{u}\right)$; and (iv) both the wheels lose contact with the ground $\left(z_{u L}-z_{0 L} \geq \delta_{u}\right.$ and $\left.z_{u R^{-}} z_{0 L} \geq \delta_{u}\right)$. The corresponding force components are illustrated in the Appendix II.

\subsection{Asymmetric Damper Modeling}

The left- and right suspension damping forces, $f_{d s k}$ and $f_{d u k}$ acting on the sprung and unsprung masses, respectively, and $T_{d s k}$, the torque due to the damper forces acting on the sprung mass in Eq (13), when equivalent linear damping coefficient is considered, are obtained from:

$$
f_{d s k}=C_{\mathrm{s}} \Delta \dot{l}_{k} \frac{\partial\left(\Delta \dot{l}_{k}\right)}{\partial \dot{z}_{\mathrm{s}}} \quad f_{d u k}=C_{s} \Delta \dot{l}_{k} \frac{\partial\left(\Delta \dot{l}_{k}\right)}{\partial \dot{z}_{u k}} \quad ; \text { and } T_{d s k}=C_{\mathrm{s}} \Delta \dot{l}_{k} \frac{\partial\left(\Delta \dot{l}_{k}\right)}{\partial \dot{\phi}_{\mathrm{s}}} \quad k=R, L
$$

where a dot over $\Delta l$ denotes the suspension deflection rate.

Influences of suspension damping asymmetry on the kinematic and dynamic responses of the proposed half-car model are evaluated by considering asymmetric viscous damping forces acting on the sprung and unsprung masses $\left(f_{d s k}, f_{d u k}\right)$. The damping forces are described through a bilinear force-velocity model [Verros et al., 2000] and the forces in compression and rebound, and the corresponding moments imposed on the sprung mass, $T_{d s k-c}$ and $T_{d s k-r}(k=R, L)$ are formulated considering $C_{c}$ as the compression damping coefficient, $\rho$ as the damping asymmetry ratio $(\rho=$ Ratio of rebound to compression damping coefficient), such that: 


$$
\begin{aligned}
& C_{c} \Delta i_{k} \frac{\partial\left(\Delta \dot{l}_{k}\right)}{\partial \dot{z}_{s}} \quad \Delta i_{k} \leq 0 \\
& f_{d s k}=\left\{\begin{array}{cc}
\partial \dot{z}_{s} & k=R, L
\end{array}\right.
\end{aligned}
$$

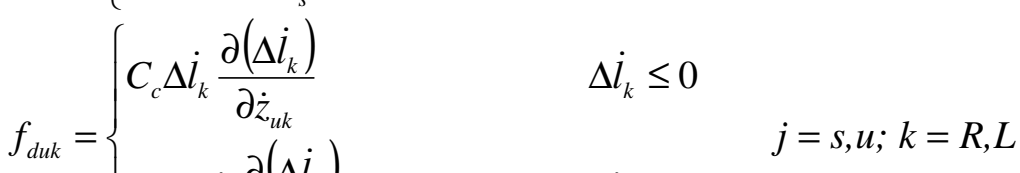

$$
\begin{aligned}
& \rho C_{c} \Delta i_{k} \frac{\partial\left(\Delta \dot{l}_{k}\right)}{\partial \dot{z}_{u k}} \quad \Delta \dot{l}_{k}>0 \\
& T_{d s k}= \begin{cases}C_{c} \Delta \dot{l}_{k} \frac{\partial\left(\Delta \dot{l}_{k}\right)}{\partial \dot{\phi}_{s}} & \Delta i_{k} \leq 0 \\
& k=R, L\end{cases} \\
& \rho C_{c} \Delta i_{k} \frac{\partial\left(\Delta \dot{l}_{k}\right)}{\partial \dot{\phi}_{s}} \quad \Delta i_{k}>0
\end{aligned}
$$

The asymmetric viscous damping forces acting on the sprung and unsprung masses, and the corresponding moments imposed on the forces on the sprung mass, as obtained from Eq (18), are employed in Eq (13) to attain the solution when an asymmetric damper is considered in the model.

\subsection{Performance Analysis Method}

The equations of motion of the proposed kineto-dynamic roll plane model as given in Eq (13) are solved to evaluate the influences of damper asymmetry on the kineto-dynamic response measures corresponding to ride and handling performances of the vehicle. The responses of the proposed roll-plane model are evaluated under transient vertical excitations representing idealized bump and potholes defining the inputs at the right wheel. The bump excitation is synthesized by a rounded pulse displacement, given by [Rakheja and Sankar, 1985, Balike et al., 2011 (ref 29)]:

$$
z_{0}(t)=z_{0 \max } \frac{e^{2}}{4}\left(\frac{2 V t}{x_{d}}\right)^{2}(e)^{-2 V t / x_{d}}
$$

where $z_{\text {Omax }}$ is the maximum amplitude, $e=2.71828, x_{d}$ is the distance from the beginning of the bump at which peak amplitude occurs and $V$ is the vehicle forward velocity. The above formulation is also applied to synthesize a negative displacement, idealizing a pothole input, by letting $z_{0 \max }<0$. In this study, $z_{0 \max }$ and $x_{d}$ are considered to be $50 \mathrm{~mm}$ and $0.4 \mathrm{~m}$, respectively. The responses of the model under above defined bump and pothole excitations are evaluated in terms of sprung mass vertical acceleration and roll angle, normalized load transfer, defined as the ratio of load transfer to the total load, and left- and right wheel camber angle variations.

The handling performance of the model with asymmetric damper are evaluated under a lateral acceleration input induced by a steady steering maneuver or crosswinds, approximated by a rounded-step function [Su, 1990], such that: 
$a_{y}(t)=A_{\max }\left[1-e^{-\sigma t}(1+\sigma t)\right.$

where $a_{y}$ is the effective lateral acceleration, $A_{\max }$ is the peak magnitude of acceleration and $\sigma$ is the parameter describing the slope of the lateral acceleration before reaching steady-state value. The responses of the model under lateral acceleration excitation are evaluated in terms of sprung mass roll angle and roll rate, and left- and right wheel camber angle variations.

Two types of asymmetric dampers were selected for the relative analyses: (a) $\zeta_{c}=0.1$ and $\rho=5$; and (b) $\zeta_{c}=0.2$ and $\rho=2$, where $\zeta_{c}$ denotes the compression mode damping ratio $\left(\zeta_{c}=\right.$ $C_{c r i t} C_{c}$ ). The responses of the kineto-dynamic roll-plane model with asymmetric dampers are compared with those of the model with a linear equivalent damper. The equivalent linear damper is realized assuming dissipated energy similarity, such that linear damping coefficient, $C_{s}=\zeta_{c} C_{c r i t}(1+\rho) / 2$ [Milliken and Milliken, 1995, Rajalingham and Rakheja, 2003], where $C_{c r i t}$ is the critical damping ratio of the model. The model parameters used in simulation are summarized in Table 1.

Table 1: Vehicle and suspension data [Balike et al., 2010 (ref 30)]

\begin{tabular}{l|l}
\hline Parameter & Value \\
\hline Sprung mass $\left(m_{s}\right)$ & $878.76 \mathrm{~kg}$ \\
Sprung mass moment of inertia about $c g\left(I_{x}\right)$ & $247.00 \mathrm{~kg}-\mathrm{m}^{2}$ \\
Unsprung mass $\left(m_{u R}\right.$ and $\left.m_{u L}\right)$ & $42.27 \mathrm{~kg}$ \\
Unsprung mass moment of inertia about $x$ - axis $\left(I_{u x}\right)$ & $1.86 \mathrm{~kg}-\mathrm{m}^{2}$ \\
Suspension spring stiffness $\left(K_{s}\right)$ & $38404 \mathrm{~N} / \mathrm{m}$ \\
Suspension damping rate $\left(C_{s}\right)$ & $3593.4 \mathrm{Ns} / \mathrm{m}$ \\
Tire vertical stiffness $\left(K_{t}\right)$ & $200 \mathrm{kN} / \mathrm{m}$ \\
Tire damping rate $\left(C_{t}\right)$ & $352.27 \mathrm{Ns} / \mathrm{m}$ \\
Tire lateral stiffness $\left(K_{t l}\right)$ & $100 \mathrm{kN} / \mathrm{m}$ \\
Tire effective radius $(R)$ & $0.35 \mathrm{~m}$ \\
Torsion bar stiffness $\left(K_{t b}\right)$ & $560 \mathrm{Nm}-\mathrm{rad}$ \\
\hline
\end{tabular}

\section{Results and Discussion}

\subsection{Kineto-dynamic Ride Performance Evaluation}

The responses of the model with asymmetric damping under $50 \mathrm{~mm}$ bump and pothole inputs at a forward velocity of $3 \mathrm{~m} / \mathrm{s}$ are compared with those of the model with equivalent linear damper in Figs. 3 (a) and (b), while the camber angle responses of the left-and right wheels under these excitations are illustrated in Figs. 4 (a) and (b). The kineto-dynamic model with light compression damping $\left(\zeta_{c}=0.1, \rho=5\right)$ yields lower peak sprung mass acceleration and roll angle response of the model compared with the other dampers. Both the peak acceleration and roll responses of the same damper, however, are relatively higher under the pothole input, as seen in Figs. 3(a) and (b). The peak sprung mass response of the model with linear and bilinear dampers with compression mode damping ratios of 0.2 and 0.1 , respectively, are $2.2,1.8$ and $1.5 \mathrm{~m} / \mathrm{s}^{2}$, under the bump input, and $-2.25,-2.5$ and $-2.7 \mathrm{~m} / \mathrm{s}^{2}$ under the pothole input. The peak roll responses of the model with linear and bilinear dampers with compression mode damping ratios of 0.2 and 0.1 , respectively, are $-2.2,-2$ and $-1.8^{\circ}$ under the bump input, and $2.2,2.55$, and $3^{\circ}$ under the pothole input. The results in the figure thus suggest that the roll angle 
responses of the model with bilinear dampers are opposite under the bump and pothole inputs. The model with lower compression mode damping $\left(\zeta_{c}=0.1\right)$ yields significantly larger roll angle response compared to those of the model with linear and bilinear damper $\left(\zeta_{c}=0.2\right)$ under the pothole type input. It is thus evident that the damper synthesis demands an additional design compromise in terms of conflicting roll angle response under bump and pothole excitations.

Figure 3: Comparisons of sprung mass responses of the kineto-dynamic model with bilinear $\left(\zeta_{c}=0.1 ; \rho=5\right.$ and $\left.\zeta_{c}=0.2 ; \rho=2\right)$ and linear equivalent dampers under idealized bump and pothole type excitations $\left(z_{0 \max }= \pm 50 \mathrm{~mm}\right)$ : (a) vertical acceleration; and (b) roll angle $(\mathrm{V}=3 \mathrm{~m} / \mathrm{s})$.

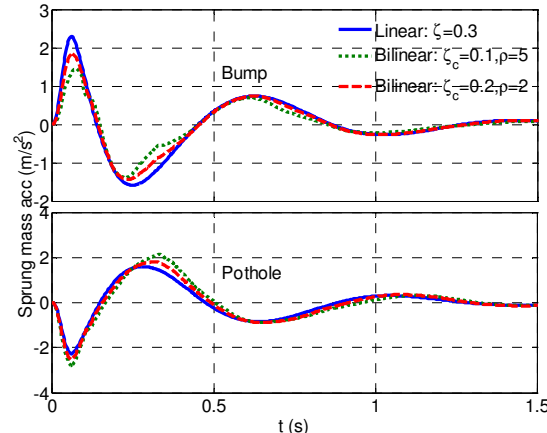

(a)

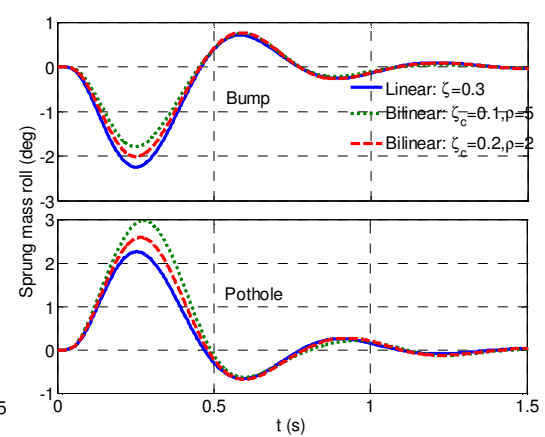

(b)

Damping asymmetry also yields important influence on the camber angle variation responses of the suspension, particularly that of the left wheel (when excitation is given to the right wheel), as shown in the Figs. 4 (a) and (b). The peak camber variations of the unexcited wheel (left wheel) are $2.1,1.9$ and $1.5^{\circ}$, respectively, under the bump excitation with the linear and bilinear damper with compression damping ratios of 0.2 and 0.1. Under the pothole input, the kineto-dynamic model with linear damper exhibits considerably smaller left wheel camber variation response compared to those of the model with bilinear damper. The influence of damper asymmetry on the camber variation response of the right wheel (excited wheel), on the other hand, is less significant, as seen in the Fig. 4 (b). It should be noted that the camber responses shown in the figures are the net results of bump and roll cambers, and the excited wheel experiences both the bump and roll, while the unexcited wheel experiences only the roll camber. This clearly suggests that the asymmetric damping could influence roll camber response of a suspension apart from the bump camber, as observed in [Balike et al. 2010 (ref 3) and Balike et al. 2011 (ref 4)]. Such a coupling between the roll camber and the damper asymmetry has not been identified in the reported studies.

The results in Figs. 3 and 4 show significant influences of asymmetric damping on the kinematic and dynamic responses of the roll-plane vehicle model, while the results are limited to a very low vehicle speed $(3 \mathrm{~m} / \mathrm{s})$. The influences of damper asymmetry on the responses are thus further investigated over a wide range of forward speeds ( 3 to $15 \mathrm{~m} / \mathrm{s}$ ). Figures 5 to 7 illustrate the peak magnitudes of kinematic and dynamic responses of the model with three different dampers as a function of vehicle forward velocity. 
Figure 4: Comparisons of camber angle variations of the kineto-dynamic model with bilinear $\left(\zeta_{c}=0.1 ; \rho=5\right.$ and $\left.\zeta_{c}=0.2 ; \rho=2\right)$ and linear equivalent dampers to idealized bump and pothole type excitations $\left(z_{0 \max }= \pm 50 \mathrm{~mm}\right)$ : of (a) the left wheel; and (b) the right wheel.

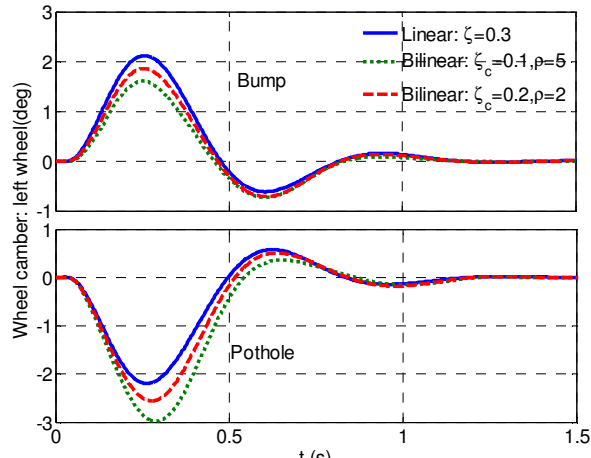

(a)

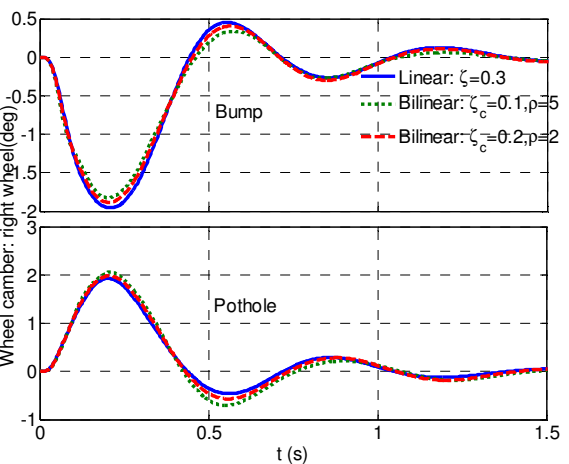

(b)

The peak sprung mass acceleration and roll angle responses of the model with linear and bilinear dampers are compared in Figs. 5 (a) and (b), respectively, as a function of the speed. Under the bump input, the bilinear damper with lower compression mode damping $\left(\zeta_{c}=0.1 ; \rho=5\right)$ yields lowest peak acceleration response at speeds below $7 \mathrm{~m} / \mathrm{s}$ and lowest roll angle at speeds below $12 \mathrm{~m} / \mathrm{s}$. The increase in peak acceleration at higher velocities is attributable to increase in the second peak rather than the first peak response. A similar trend was also observed in the second peak in roll angle response of the model with $\zeta_{c}=0.1$ under bump input at speeds above $12 \mathrm{~m} / \mathrm{s}$. The model with equivalent linear damper yields highest acceleration at speeds below $10 \mathrm{~m} / \mathrm{s}$ and highest roll angle in the entire speed range. The bilinear damper with $\zeta_{c}=0.2$ yields lowest peak sprung mass acceleration and roll angle response to bump inputs at speeds above $7 \mathrm{~m} / \mathrm{s}$. Under the pothole input, linear damper yields lowest peak acceleration and roll angle responses in the entire speed range. The results suggest conflicting design demands on the damper synthesis and that a bilinear damper with $\zeta_{c}=0.2$ could yield good compromises in responses to bump and pothole excitations.

Figure 5: Comparisons of sprung mass responses of kineto-dynamic model with bilinear $\left(\zeta_{c}=0.1 ; \rho=5\right.$ and $\left.\zeta_{c}=0.2 ; \rho=2\right)$ and linear equivalent dampers under idealized bump and pothole type excitations $\left(z_{0 \max }= \pm 50 \mathrm{~mm}\right)$ in the forward velocity range 3 to $15 \mathrm{~m} / \mathrm{s}$ : (a) vertical acceleration; and (b) roll angle.

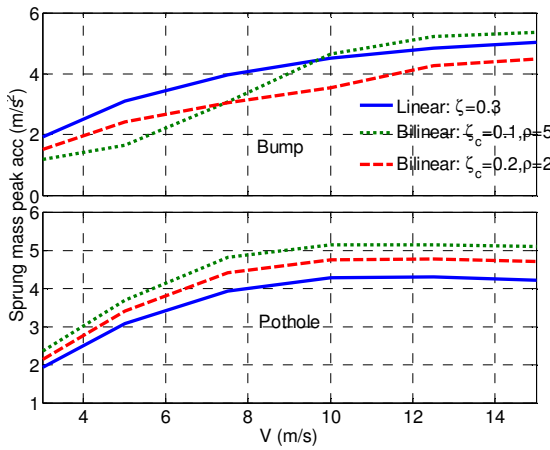

(a)

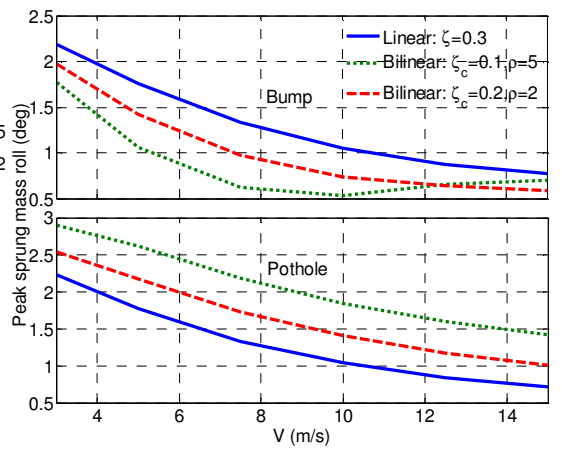

(b) 
The camber angle variation responses of the left- and right wheels of the kinetodynamic roll-plane model with linear and bilinear dampers under bump and pothole inputs, as function of forward velocity are presented in Figs. 6 (a) and (b), respectively. The peak left wheel camber angle response, which is mainly due to contribution of the roll camber angle, exhibits trend similar to the peak roll angle response, as shown in Fig. 5 (b). Under the bump input, higher peak camber angle of the left wheel is observed for the model with linear dampers until the speed of $12.5 \mathrm{~m} / \mathrm{s}$, while above this speed, the model with $\zeta_{c}=0.1$ yields higher peak camber responses. The left wheel camber variation under the pothole input is more uniform, with the linear damper yielding the lowest camber variation in the entire velocity range, as seen in Fig. 6 (a). The peak camber angle response exhibited by the right wheel of the kineto-dynamic model with bilinear damper of $\zeta_{c}=0.2$ is the lower above $5 \mathrm{~m} / \mathrm{s}$ compared to those with other dampers under bump excitation. At speeds below $7 \mathrm{~m} / \mathrm{s}$, the kineto-dynamic model with linear damper yields higher right wheel camber response under pothole response, which is attributed to the higher roll angle at lower velocities. The bump camber which is opposite in direction to that of the roll camber reduces the net camber response (compared to the left wheel camber responses). At high velocity bump and pothole inputs, however, the contribution of roll camber is higher, as compared to that of bump camber in the camber variation response. The results thus suggest that damping asymmetry causes difference in the camber angle response under dynamic events like bump excitations.

Figure 6: Comparisons of camber angle variation responses of kineto-dynamic model with bilinear $\left(\zeta_{c}=0.1 ; \rho=5\right.$ and $\left.\zeta_{c}=0.2 ; \rho=2\right)$ and linear equivalent dampers to idealized bump and pothole type excitations $\left(z_{0 \max }= \pm 50 \mathrm{~mm}\right)$, in forward velocity range 3 to $15 \mathrm{~m} / \mathrm{s}$ : (a) the left wheel; and (b) the right wheel.

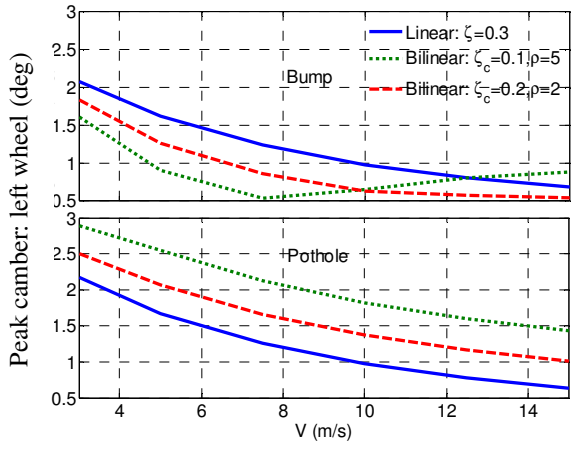

(a)

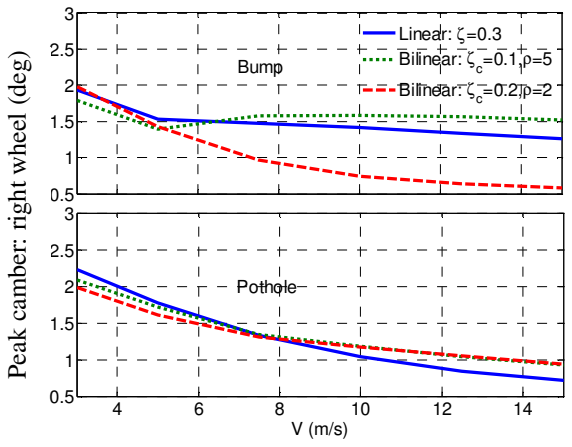

(b)

Lower compression mode damping yields slightly lower normalized load transfer response of the model at low velocity $(3-5 \mathrm{~m} / \mathrm{s})$ bump inputs, while the load transfer response of the model with the same damper at velocities above $5 \mathrm{~m} / \mathrm{s}$ is considerably larger compared to those with the other dampers, as seen in Fig 7. Furthermore, under bump excitations, the bilinear dampers with $\zeta_{c}=0.1$ cause wheel lift-off (normalized load transfer=1) at a relatively lower speed of $10 \mathrm{~m} / \mathrm{s}$, while the bilinear dampers with $\zeta_{c}=0.2$ and the linear dampers yield wheel lift-off at relatively higher speed of 12.5 and $15 \mathrm{~m} / \mathrm{s}$, respectively. Wheel lift-off of the model under pothole input, in general, occurs at much 
lower velocities than those observed under the bump inputs, irrespective of the type of damper employed. The bilinear damper with $\zeta_{c}=0.1$ yields normalized load transfer of 1 at $7.5 \mathrm{~m} / \mathrm{s}$, while the linear and higher compression damping bilinear dampers yield wheel lift-off at only slightly higher speeds. Ironically, linear damper yields better (lower) load transfer response under both bump and pothole inputs in the entire velocity range.

Figure 7: Comparisons of normalized load transfer response of kineto-dynamic model with bilinear $\left(\zeta_{c}=0.1 ; \rho=5\right.$ and $\left.\zeta_{c}=0.2 ; \rho=2\right)$ and linear equivalent dampers under idealized bump and pothole type excitations $\left(z_{0 \max }= \pm 50 \mathrm{~mm}\right)$, in the forward velocity range 3 to $15 \mathrm{~m} / \mathrm{s}$.

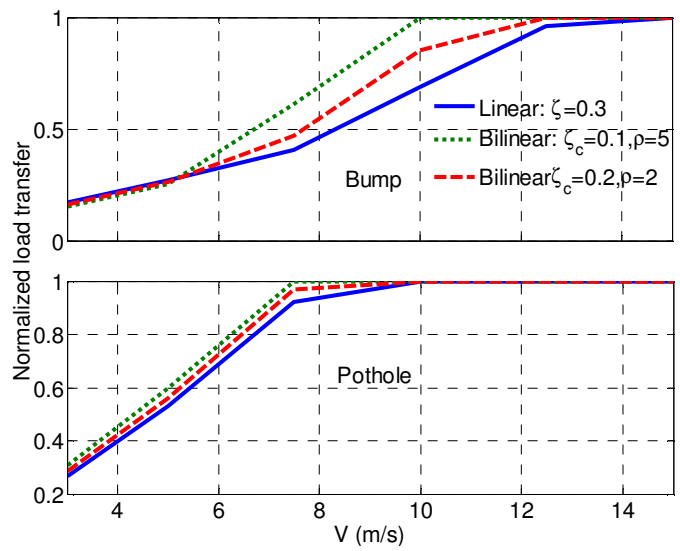

The results in Figs. 3 to 7 thus suggest significant influences of asymmetric dampers on the dynamic and kinematic response measures under road vertical excitations, which are also complex functions of the vehicle forward velocity and the type of input. Synthesis of an asymmetric damper would also necessitate consideration of the kinematic and dynamic responses of the kineto-dynamic half-car model under different inputs including random road excitations. The evaluation under random excitations, however, is beyond the scope of this study.

\subsection{Handling Performance Evaluations}

The responses of the proposed model are further evaluated under rounded-step lateral acceleration excitation, as described in Eq (20), of $6 \mathrm{~m} / \mathrm{s}^{2}$ magnitude in order to study the handling performance of the vehicle with suspension dampers of different rebound to compression asymmetry ratios, and are illustrated in Figs. 8 and 9. The peak sprung mass roll angle and roll rate responses of the model with a low compression mode damping $\left(\zeta_{c}=0.1\right)$ are higher as compared to those with a linear damper or with an asymmetric damper of $\zeta_{c}=0.2$, as seen in Figs 8 (a) and (b). The roll angle response of the model with $\zeta_{c}=0.1$ is near $4.75^{\circ}$, which is 5 and $8 \%$ more than those with bilinear damper of $\zeta_{c}=0.2$ and the linear damper, respectively. The roll rate responses seem somewhat less sensitive to variations in the damping asymmetry ratio (Fig 8 (b)). This can be attributed to the fact that all the three dampers considered in this study are equivalent, as far as the effective damping is concerned. It needs to be noted that the variations in the roll angle and roll 
rate responses are due to net damping from left and right suspensions, with one being in compression and other in extension. Moreover, the observed oscillations occur about intermediate points in compression and extension of the suspension. The degree of influence of the damper asymmetry would thus be dependent upon these intermediate positions, which are related to the magnitude of lateral acceleration excitation. The results in Fig. 8 suggest that a linear damper yields better roll damping under lateral acceleration inputs, as compared to that with an asymmetric damper.

Figure 8: Comparisons of sprung mass responses of the kineto-dynamic model with bilinear $\left(\zeta_{c}=0.1 ; \rho=5\right.$ and $\left.\zeta_{c}=0.2 ; \rho=2\right)$ and linear equivalent dampers under a $6 \mathrm{~m} / \mathrm{s}^{2}$ rounded-step lateral acceleration excitation: (a) roll angle; and (b) roll rate.

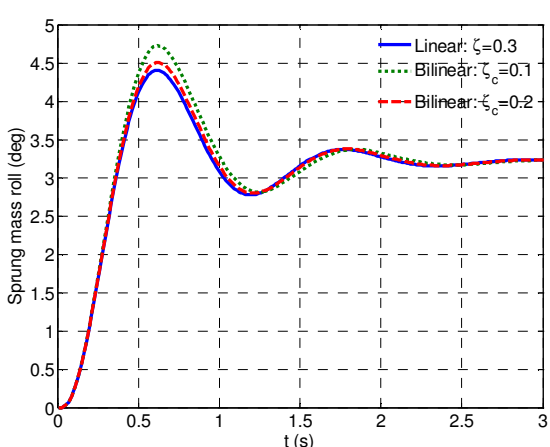

(a)

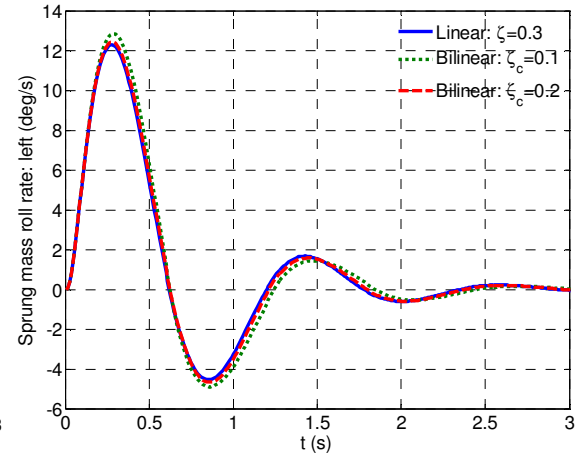

(b)

Figures 9 (a) and (b) illustrate the camber angle response of the left and right wheels to the rounded-step lateral acceleration input. The left and right wheels correspond to the inner and outer wheels, respectively, of the vehicle negotiating a turn. The damping asymmetry, as in the case of pothole type excitation, affects on the camber angle variation responses of the model. The peak roll camber variations of the inner wheel (left wheel) are $-3.7,-3.85$ and $-4.1^{\circ}$, respectively, with the linear and bilinear damper with compression damping ratios of 0.2 and 0.1 . The influence of damper asymmetry on the roll camber variation response of the outer wheel, on the other hand, is less significant, as seen in the Fig. 9 (b). It has been already shown in Figs. 4 (a) and (b) that the roll camber response of the model is influenced by the considered variations of the rebound to compression damping asymmetry. The results in Fig. 8 and 9 further suggest that a very low damping is not desirable from the perspectives of handling performance measures considered in this study.

The results of this study clearly suggest that the kinematic response measures such as camber variations, which are generally considered as pure kinematic phenomena necessitate consideration during the dynamic analysis. The study has further shown that the proposed model can effectively be employed to study the ride and handling performance measures of the vehicle. Although the simulation results from the proposed model have yielded important guidelines, validation of the model responses with experimental data prior to damper synthesis is essential. Formulation of a performance index comprising conflicting performance measures and subsequent solution of the optimization problem would be vital for the synthesis of an asymmetric damper. An optimal damper synthesis also necessitates consideration of high velocity saturation of the damper and extended vehicle models to incorporate pitch mode motion of the sprung mass. 
Figure 9: Comparisons of camber angle variations of the kineto-dynamic model with bilinear $\left(\zeta_{c}=0.1 ; \rho=5\right.$ and $\left.\zeta_{c}=0.2 ; \rho=2\right)$ and linear equivalent dampers under rounded-step lateral excitation: of (a) the left wheel; and (b) the right wheel.

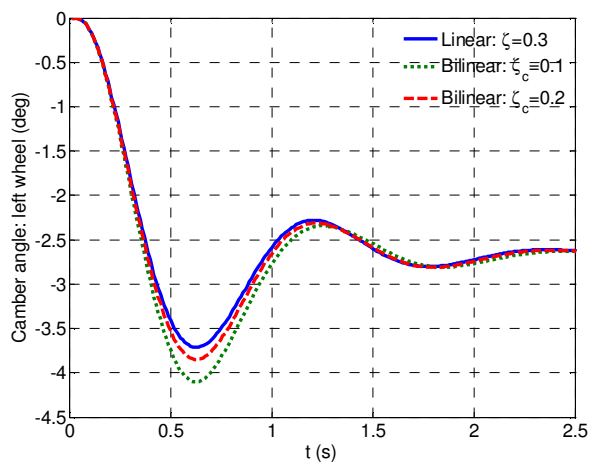

(a)

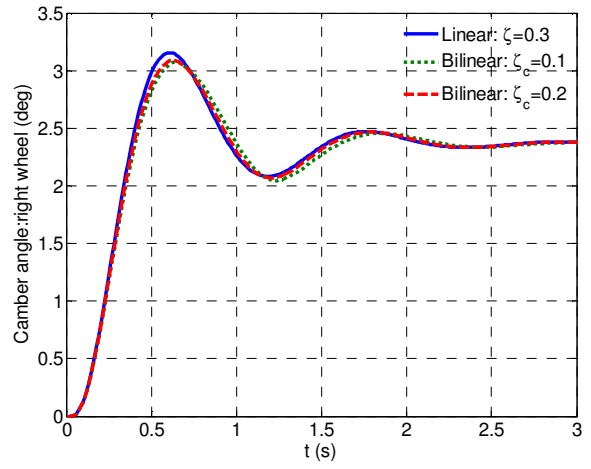

(b)

\section{Conclusions}

A kineto-dynamic roll-plane model of a vehicle incorporating linkage kinematics of a double wishbone suspension is proposed to investigate the influences of damper asymmetry on the kineto-dynamic responses of a vehicle. The proposed model is shown to be effective for studying the vehicle responses under vertical road and lateral acceleration inputs. The results of the study suggested that the responses are complex functions of damper asymmetry ratio, vehicle forward speed and type of input. Comparisons of the responses with two different asymmetric dampers of similar effective damping coefficients showed that a very low compression mode damping with a large rebound mode damping helps reduce the sprung mass vertical acceleration, roll angle and wheel camber of the unexcited wheel under a bump input. Under the pothole input, however, an opposite trend is observed in these responses. A very low compression mode damping is thus shown to be undesirable, while an asymmetric damper with compression mode damping ratio of 0.2 coupled with damping asymmetry ratio in the range of 2 would be a better design compromise. Furthermore, a very low compression mode damping is observed to increase the sprung mass roll angle and roll camber of the inner wheels under a steady lateral acceleration input. The results show that an asymmetric damper synthesis is complex task involving a large number of design compromises among the vertical and roll dynamic, and kinematic performance measures. Formulation of a performance index comprising dynamic and kinematic responses of the model, and subsequent minimization of the performance index would be instrumental in obtaining an optimal asymmetric damper.

\section{References}

1. Milliken W D and Milliken W F (1995) Race Car Dynamics, SAE Inc, Warrendale, PA

2. Dixon, J. C. (2007), The Shock Absorber Handbook, SAE Inc., Warrendale, PA. 
3. Balike, K. P., Rakheja, S. and Stiharu, I. (2010) 'Influence of automotive damper asymmetry on the kinematic and dynamic responses, and selection of optimal damper asymmetric parameters', Int. Journal of Vehicle Design, Vol. 54. No.3 pp. 191-216.

4. Balike, K. P., Rakheja, S. and Stiharu, I. (2011) 'Optimal synthesis of a two-stage asymmetric damper of an automotive suspension considering wheel camber variations', IMechE Part D, Journal of Automobile Engineering, Vol. 225, online.

5. Anderson, R. J. and Fan, Y. (1990) 'Dynamic testing and modeling of a bus shock absorber', SAE Technical Paper 902282.

6. Duym, S., Stiens, R. and Rebrouck, K. (1997) 'Evaluation of shock absorber models', Vehicle System Dynamics, Vol. 27, pp. 109-127.

7. Basso, R. (1998), 'Experimental characterization of damping force in shock absorber with constant velocity excitation', Vehicle System Dynamics, Vol. 30, pp. 431-442.

8. Gacka, S. P. and Doherty, C. G. (2006) 'Design analysis and testing of dampers for a formula SAE race car', SAE Technical Paper, 2006-01-3641.

9. Simms, A. and Crolla, D. (2002) 'The influence of damper properties on vehicle dynamic behavior', SAE Technical Paper, 2002-01-0319.

10. Gillespie, T. D (1992), Fundamentals of Vehicle Dynamics, SAE Inc., Warrendale, PA.

11. Warner, B. and Rakheja, S. (1996) 'An investigation of the influence of high performance dampers on the suspension performance of a quarter vehicle', $S A E$ Technical Paper, 962552.

12. Rajalingham, C. and Rakheja, S. (2003) 'Influence of suspension damper asymmetry on vehicle vibration response to ground excitation', Journal of Sound and Vibration, Vol. 266, pp. 1117-1129.

13. Verros, G., Natsivas, S. and Stepan, G. (2000) 'Control and dynamics of quarter-car models with dual-rate damping', Journal of Vibration and Control, Vol. 6, pp. 1045-1063.

14. Fukushima, N., Hidaka, K. and Iwata, K. (1983) 'Optimum characteristics of automotive shock absorbers under driving conditions and road surfaces', Int. J. of Vehicle Design, Vol. 4(5), pp. 463-473.

15. Gobbi, M., and Mastinu, G. (2001) 'Analytical description and optimization of the dynamic behavior of passively suspended road vehicles', J of Sound and Vibration, Vol. 245/3, pp. 457-481

16. Alkhatib, R., Jafar, G. N. and Golnaraghi, M.F. (2004) 'Optimal design of passive linear suspension using genetic algorithm', J of Sounds and Vibration, Vol. 275, pp. 665-691.

17. Verros, G., Natsiavas, S. and Papadimitriou, C. (2005) 'Design optimization of quarter-car models with passive and semi-active suspensions under random road excitation', Journal of Vibration and Control, Vol. 11, pp. 581-606.

18. Georgiou, G., Verros, G. and Natsiavas, S. (2007) 'Multi-objective optimization of quarter-car models with a passive or semi-active suspension system', Vehicle System Dynamics, Vol. 45/1, pp. 77-92.

19. Gobbi, M., Mastinu, G. and Donoselli, C. (1999) 'Optimizing a car chassis', Vehicle System Dynamics, Vol. 32, pp. 149-170. 
20. Bruulsema, I.T. and McPhee, J. J. (2002) 'Dynamic modeling and design optimization of automotive suspension systems', Proceedings of the CSME FORUM 2002, Kingston, Ontario, Canada.

21. He, Y. and McPhee, J. J. (2007) 'Application of optimization algorithms and multibody dynamics to ground vehicle suspension design', Int. Journal of Heavy Vehicle Systems, Vol. 14/2, pp. 158-192.

22. Georgiou, G. and Natsiavas, S. (2009) 'Optimal selection of suspension parameters in large scale vehicle models', Vehicle System Dynamics, Vol. 47/9, pp. 1147-1166.

23. Blundell, M. V. (1999) 'The modeling and simulation of vehicle handling Part 1: analysis method', IMechE Part K, Journal of Multibody Systems Dynamics, Vol. 213, pp. 103-118.

24. Sharp, R. S. (1991) 'Computer Codes for Road Vehicle Dynamic Models', Proceedings of Autotech 91, Brimingham, UK.

25. Su. H. (1990) An investigation of vibration isolation systems using active, semiactive and tunable passive mechanisms with application to vehicle suspensions, $\mathrm{PhD}$ Thesis, Concordia University, Canada.

26. Suh, C. H. and Radcliffe, C. W. (1978) Kinematics and Mechanisms Design, John Wiley \& Sons, New York.

27. Balike, K. P., Rakheja, S. and Stiharu, I. (2010) 'Synthesis of a vehicle suspension with constrained lateral space using roll-plane kineto-dynamic model', Transactions of SAE, Journal of Materials and Manufacturing, Vol. 3/1, pp. 305-315.

28. Rakheja, S. and Sankar, S. (1985) 'Vibration and shock isolation performance of a semi active on-off damper', Trans. of ASME, J. of Vibration, Acoustics, Stress, and Reliability in Design, Vol. 107, pp. 398-403.

29. Balike, K. P., Rakheja, S. and Stiharu, I. (2011) 'Development of kineto-dyanmic quarter- car model for synthesis of a double wishbone suspension', Vehicle System Dynamics, Vol 49, No 1-2, pp 107-128.

30. Balike, K. P., (2010), 'Kineto-dynamic analysis of vehicle suspension for optimal synthesis', Ph D thesis, Concordia University, Canada.

\section{Nomenclature}

$a_{11}=a_{22}=$ Components of chassis displacement matrix

$a_{11 k}=a_{22 k}=$ Components of wheel spindle displacement matrix

$a_{y}=$ Effective lateral acceleration

$f_{d s k}, f_{d u k}=$ Right- and left- damping forces acting on the sprung and unsprung masses

$f_{t l s k}, f_{t l u k}=$ Vertical tire forces acting on the sprung and unsprung masses

$f_{t k}=$ Tire forces

$f_{t b s,} f_{t b u k}=$ Forces transmitted to the sprung and unsprung masses

$f_{s s k}, f_{\text {suk }}(k=R, L)=$ Right- and left- suspension spring forces acting on the sprung and unsprung masses

$k=$ Subscript used to denote suspension side 'left' (L) or 'right' (R)

$l_{s 0}=$ Initial strut length

$l_{M N k}$ and $l_{O P k}=$ Upper and lower control arm lengths (left and right suspensions)

$l_{O A}, l_{O P}=$ Distances of the joints $A_{R}$ and $P_{R}$ from point $O_{R}$ (or $A_{L}$ and $P_{L}$ from point $O_{L}$ )

$m_{s}, m_{u k}=$ sprung, left and right unsprung masses 
$x_{d}=$ Distance from the beginning of the bump

$y_{u k}=$ Wheel center lateral displacement

$z_{s}, z_{u k}=$ Sprung mass and unsprung mass vertical displacements

$z_{0}=$ Road vertical input

$A_{\max }=$ Lateral acceleration amplitude

$A_{k y}, A_{k z}, B_{k y}, B_{k z}=$ Instantaneous $y$ - and $z$ - coordinates of lower and upper mounts of left and right suspensions.

$C_{s}, C_{t}=$ Suspension and tire damping coefficients

$C_{c r i t}=$ Critical damping coefficient

$F_{y}=$ Lateral force

$I_{x,} I_{u k x}=$ Mass moment of inertia of sprung and unsprung masses about $x$ - axis

$K_{s}, K_{t}, K_{t l}=$ Suspension spring, tire vertical and lateral stiffness coefficients

$L_{T}=$ Effective length of the torsion bar between attachment points $T s_{R}$ and $T c_{R}$

$M_{k}, O_{k}, N_{k} P_{k}$ and $C_{k}=$ Instantaneous coordinates of left and right suspensions joints and wheel centers

$R_{k}=$ Effective radius of wheel $k$

$R c=$ Roll centre height

$T_{C k}, T_{S k}=$ Torsion bar attachment points at chassis

$T, U, D=$ kinetic, potential and dissipative energy

$T_{s s k}, T_{d s k}=$ Moments due to right- and left- suspension spring and damping forces

$T_{t l s k}=$ Moments imposed on the sprung mass due to the right- and left- tire lateral compliance

$T_{t b s}=$ Torque transmitted to the sprung mass due to the torsion bar

$V=$ Vehicle forward velocity

$\rho=$ Ratio of rebound to compression damping coefficient, damping asymmetry ratio.

$\zeta_{c}=$ Compression mode damping ratio

$\phi_{s}=$ Sprung mass roll angle motion

$\phi_{L}, \phi_{R}=$ Left and right camber angles

$\Delta l_{k}=$ Left and right strut deflections

$\theta_{T}=$ Torsion bar twisting angle angle

$\Delta \mathrm{z}_{k}=$ Tire deflection 


\section{Appendix I}

The expressions for the kinematic responses of the left- and right ( $k=L$ and $R$ ) suspensions, which include the instantaneous coordinates of the joints and the wheel camber angles, in terms of the generalized coordinates:

$$
\begin{aligned}
& \phi_{k}=\frac{1}{D_{k}}\left[\begin{array}{l}
\left(e_{k}-2 M_{j y}\right)\left[x_{2 k}-\left(g_{k} O_{k y}+h_{k} O_{k z}\right)-\left(h_{k}-2 O_{k z}\right)\left(P_{k z 0}+z_{u k}\right)\right] \\
+\left(a_{k}-c_{k}\right)\left(g_{k}-2 O_{k y}\right)\left(e_{k}-2 M_{k y}\right) \\
-\left(g_{k}-2 O_{k y}\right)\left[x_{1 k}-\left(e_{k} M_{k y}+f_{k} M_{k z}\right)-\left(f_{k}-2 M_{k z}\right)\left(N_{k z 0}+z_{u k}\right)\right]
\end{array}\right]
\end{aligned}
$$

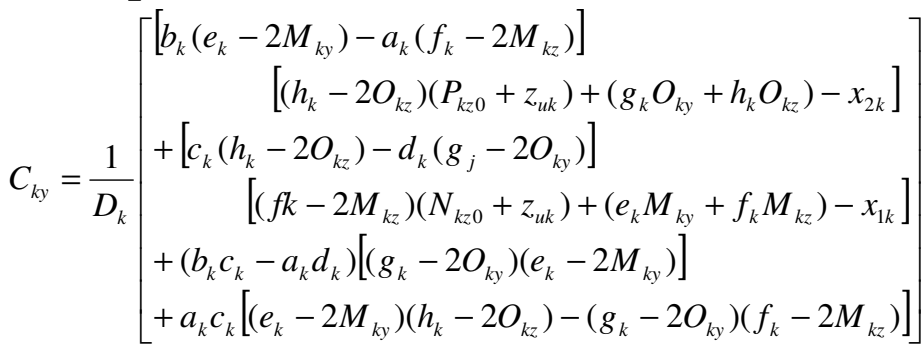

$$
\begin{aligned}
& N_{k y}=\frac{1}{D_{k}}\left[\begin{array}{l}
{\left[\left(b_{k}-d_{k}\right)\left(g_{k}-2 O_{k y}\right)+c_{k}\left(h_{k}-2 O_{k z}\right)\right]\left[\begin{array}{l}
\left(f_{k}-2 M_{k z}\right)\left(N_{k z 0}+z_{u k}\right) \\
+e_{k} M_{k y}+f_{k} M_{k z}-x_{1 k}
\end{array}\right]} \\
+a_{k}\left(f_{k}-2 M_{k z}\right)\left[\begin{array}{l}
\left(a_{k}-c_{k}\right)\left(g_{k}-2 O_{k y}\right)+x_{2 k}-\left(g_{k} O_{k y}+h_{k} O_{k z}\right) \\
-\left(h_{k}-2 O_{k z}\right)\left(P_{k z 0}+z_{u k}\right)
\end{array}\right]
\end{array}\right] \\
& N_{k z}=\frac{1}{D_{k}}\left[\begin{array}{l}
{\left[\left(d_{k}-b_{k}\right)\left(g_{k}-2 O_{k y}\right)-c_{k}\left(h_{k}-2 O_{k z}\right)\right]\left(e_{k}-2 M_{k y}\right)\left(N_{k z 0}+z_{u k}\right)} \\
+a_{k}\left[\left(h_{k}-2 O_{k z}\right)\left(P_{k z 0}+z_{u k}\right)+\left(g_{k} O_{k y}+h_{k} O_{k z}\right)-x_{2 k}\right]\left(e_{k}-2 M_{k y}\right) \\
-a_{k}\left[e_{k} M_{k y}+f_{k} M_{k z}+\left(a_{k}-c_{k}\right)\left(e_{k}-2 M_{k y}\right)-x_{1 k}\right]\left(g_{k}-2 O_{k y}\right)
\end{array}\right] \\
& P_{k y}=\frac{1}{D_{k}}\left[\begin{array}{l}
{\left[\left(b_{k}-d_{k}\right)\left(e_{k}-2 M_{k y}\right)+a_{k}\left(f_{k}-2 M_{k z}\right)\left[\begin{array}{l}
\left(h_{k}-2 O_{k z}\right)\left(P_{k z 0}+z_{u k}\right) \\
+g_{k} O_{k y}+h_{k} O_{k z}-x_{2 k}
\end{array}\right]\right.} \\
-c_{k}\left(h_{k}-2 O_{k z}\right)\left[\begin{array}{l}
\left(c_{k}-a_{k}\right)\left(e_{k}-2 M_{k y}\right)-e_{k} M_{k y}-f_{k} M_{k z} \\
+x_{1 k}-\left(f_{k}-2 M_{k z}\right)\left(N_{k z 0}+z_{u k}\right)
\end{array}\right]
\end{array}\right] \\
& P_{k z}=\frac{1}{D_{k}}\left[\begin{array}{l}
{\left[\left(d_{k}-b_{k}\right)\left(e_{k}-2 M_{k y}\right)+a_{k}\left(f_{k}-2 M_{k z}\right)\right]\left(g_{k}-2 O_{k y}\right)\left(P_{k z 0}+z_{u k}\right)} \\
-c_{k}\left[\left(f_{k}-2 M_{k z}\right)\left(N_{k z 0}+z_{u k}\right)+e_{k} M_{k y}+f_{k} M_{k z}-x_{1 k}\right]\left(g_{k}-2 O_{k y}\right) \\
-c_{k}\left[x_{2 k}-g_{k} O_{k y}-h_{k} O_{k z}-\left(c_{k}-a_{k}\right)\left(g_{k}-2 O_{k y}\right)\right]\left(e_{k}-2 M_{k y}\right)
\end{array}\right]
\end{aligned}
$$

where $a_{k}=N_{k y 0^{-}} C_{k y 0} ; b_{k}=N_{k z 0^{-}} C_{k z 0} ; c_{k}=P_{k y 0^{-}} C_{k y 0} ; d_{k}=P_{k z 0^{-}} C_{k z 0} ; e_{k}=N_{k y 0}+M_{k y 0} ; f_{k}=N_{k z 0}+M_{k z 0}$; $g_{k}=P_{k y 0}+O_{k y 0} ; \quad h_{k}=P_{k z 0}+O_{k z 0} ; \quad x_{l k}=N_{k y 0}^{2}+M_{k y 0}^{2}+N_{k z 0}^{2}+M_{k z 0}^{2} ; \quad x_{2 k} \quad=$ $P_{k y 0}^{2}+O_{k y 0}^{2}+P_{k z 0}^{2}+O_{k z 0}^{2} ;$ and $D_{k}=\left[a_{k}\left(f_{k}-2 M_{k z}\right)-b_{k}\left(e_{k}-2 M_{k y}\right)\right]\left(g_{k}-2 O_{k y}\right)$ $+\left[d_{k}\left(g_{k}-2 O_{k y}\right)-c_{k}\left(h_{k}-2 O_{k z}\right)\right]\left(e_{k}-2 M_{k y}\right)$ 


\section{Appendix II}

The forces due to tire damping, and the lateral and vertical compliance are formulated considering four different possible conditions; namely: (i) both the tires are in contact with the ground $\left(z_{u L}-z_{0 L}<\delta_{u}\right.$ and $\left.z_{u R^{-}} z_{0 R}<\delta_{u}\right)$, where $\delta_{u}$ is the static tire deflection; (ii) left wheel in contact with the ground, while the right wheel loses the ground contact $\left(z_{u L^{-}}\right.$ $z_{O L}<\delta_{u}$ and $z_{u R^{-}} z_{O R} \geq \delta_{u}$ ); (iii) right wheel is in contact with the ground, while the left wheel loses the ground contact $\left(z_{u L}-z_{O L} \geq \delta_{u}\right.$ and $\left.z_{u R^{-}} z_{O R}<\delta_{u}\right)$; and (iv) both the wheels lose contact with the ground $\left(z_{u L}-z_{0 L} \geq \delta_{u}\right.$ and $\left.z_{u R^{-}} z_{0 L} \geq \delta_{u}\right)$. These are summarized below:

$$
\begin{aligned}
& f_{t l s k}=K_{t l}\left(y_{u k}-\phi_{k} R_{k}\right) \frac{\partial\left(y_{u k}-\phi_{k} R_{k}\right)}{\partial z_{\mathrm{s}}} \text {; } \\
& f_{t l u k}=K_{t l}\left(y_{u k}-\phi_{k} R_{k}\right) \frac{\partial\left(y_{u k}-\phi_{k} R_{k}\right)}{\partial z_{u k}} \\
& T_{t l s k}=K_{t l}\left(y_{u k}-\phi_{k} R_{k}\right) \frac{\partial\left(y_{u k}-\phi_{k} R_{k}\right)}{\partial \phi_{s}} ; \text { and } f_{t k}=K_{t} \Delta z_{t k}+C_{t} \Delta \dot{z}_{t k} ; k=R, L \\
& \text { for } z_{u L} z_{0 L}<\delta_{u} \text { and } z_{u R^{-}} z_{0 R}<\delta_{u} \\
& f_{t l s R}=0 ; f_{t l s L}=K_{t l}\left(y_{u L}-\phi_{L} R_{L}\right) \frac{\partial\left(y_{u L}-\phi_{L} R_{L}\right)}{\partial z_{\mathrm{s}}} \\
& T_{t l s R}=0 ; \quad T_{t l s L}=K_{t l}\left(y_{u L}-\phi_{L} R_{L}\right) \frac{\partial\left(y_{u L}-\phi_{L} R_{L}\right)}{\partial \phi_{s}} \\
& f_{\text {tluR }}=0 ; f_{\text {tluL }}=K_{t l}\left(y_{u L}-\phi_{L} R_{L}\right) \frac{\partial\left(y_{u L}-\phi_{L} R_{L}\right)}{\partial z_{u L}} \\
& f_{t R}=0 ; f_{t L}=K_{t} \Delta z_{t L}+C_{t} \Delta \dot{z}_{t L} \quad k=R, L \quad \text { for } z_{u L^{-}} z_{0 L}<\delta_{u} \text { and } z_{u R^{-}} z_{0 R} \geq \delta_{u} \\
& f_{t l s R}=K_{t l}\left(y_{u R}-\phi_{R} R_{R}\right) \frac{\partial\left(y_{u R}-\phi_{\mathrm{R}} R_{R}\right)}{\partial z_{\mathrm{s}}} ; f_{t l s L}=0 \\
& T_{t l s R}=K_{t l}\left(y_{u R}-\phi_{R} R_{R}\right) \frac{\partial\left(y_{u R}-\phi_{R} R_{R}\right)}{\partial \phi_{s}} ; \quad T_{t l s L}=0 \\
& f_{\text {tluR }}=K_{t l}\left(y_{u R}-\phi_{R} R_{R}\right) \frac{\partial\left(y_{u R}-\phi_{R} R_{R}\right)}{\partial z_{u R}} ; f_{t l u L}=0 \\
& f_{t s R}=K_{t} \Delta z_{t R}+C_{t} \Delta \dot{z}_{t R} ; \quad f_{t L}=0 \quad k=R, L \text { for } z_{u L^{-}} z_{0 L} \geq \delta_{u} \text { and } z_{u R^{-}} z_{0 R}<\delta_{u} \\
& f_{t l s k}=0 ; T_{t l s k}=0 ; \quad f_{\text {tluk }}=0 ; f_{t k}=0 \quad k=R, L \text { for } z_{u L}-z_{0 L} \geq \delta_{u} \text { and } z_{u R}-z_{0 R} \geq \delta_{u}
\end{aligned}
$$

